\title{
GEOSPATIAL MONITORING OF ENGINEERING STRUCTURES AS A PART OF BIM
}

\author{
R. Shults ${ }^{1}$ \\ ${ }^{1}$ Research Institute of Geodesy, Topography, and Cartography, v.v.i. Ustecka, 98, CZ-25066 Zdiby, Czech Republic - \\ roman.shults@vugtk.cz
}

Commission V, WG V/7

KEY WORDS: Geospatial Monitoring, BIM, Monitoring Workflow, Finite Element Model, Data Analysis.

\begin{abstract}
:
Among the various civil engineering tasks, geospatial monitoring of engineering structures takes an essential place. In the digital era, the concept of geospatial monitoring needs a total re-definition. The widespread dissemination of BIM creates new opportunities and challenges simultaneously for the monitoring problem. The presented paper aims to develop the new concept of geospatial monitoring tailored to BIM features and possibilities. The interrelationship of BIM, building life cycle, and geospatial monitoring of engineering structures has been demonstrated. The critical element of geospatial monitoring is its workflow. Up today, BIM is considered separately from monitoring workflow. However, the main stages of geospatial monitoring cannot get by without BIM. That is why the new monitoring workflow with BIM support has been suggested. The workflow takes into account three main stages: design, data collection, and analysis. For each of these stages, the role and application of BIM have been examined. The question of geospatial monitoring design has been addressed, and the new approach to monitoring design with BIM involvement has been suggested. It was pointed out the meaning of BIM for the data collection stage and especially for analysis. The refined concept of geospatial monitoring has been considered as a case study of high-rise building monitoring.
\end{abstract}

\section{INTRODUCTION}

Modern geospatial monitoring comprises many tools and different equipment. Despite the well-developed concept, it has become obsolete due to the involvement of new technologies and especially software. Today, geospatial monitoring is not just about geodetic measurements; instead, it is a more complicated problem. The issue of the integration and complex processing of geodetic and non-geodetic information provided by, e.g., extensometers, piezometers, etc., creates new challenges. This is why the aggregation of the measured displacements with the results of the structure's temperature measurements, augmented by ground humidity measurements, the stress in structures and ground is a problem, the solution of which is at the initial stage. However, the new measuring equipment is only a part of the problem. The software changes have become even more significant. From this point of view, different equipment needs different software for processing. Therefore, the person conducting geospatial monitoring encounters the big problem: various equipment, different measured values with different meanings, and different software for their processing. Of course, this concerns just data collection. But how to be with analysis of such complex data structure? That is a big challenge. The solution should be found in employing software that allows simultaneously fusing such multiple data. In this way, the solution is in the data integration using building information model (BIM) technologies. BIM is not a new concept for designers, architects, civil engineers, and surveyors. But everyone treats that concept from their own point of view. For designers and architects, BIM, first of all, is an architectural model that was created by an architect using specialized software. For civil engineers, BIM is an analytical or calculation model (primarily based on the FEM model) (Barazzetti et al. 2015, Crespi et al. 2016, Xu et al. 2019) the idealized model that reflects meaningful features of the construction under specific loads. Surveyors mostly use BIM as a source of geometric parameters that engineering structures must comply with ( $\mathrm{Li}$ et al. 2007,
Tang et al. 2010, Qin et al., 2021). Probably, such a situation is typical for supporting the construction process, but how about geospatial monitoring? For geospatial monitoring, we cannot consider the models mentioned above separately rather, it is necessary to use the benefits of their integration (Shults, 2020). It is clear that BIM has all the essential information about the structure. At the same time, it allows integrating various data and making their analysis. Moreover, if some parameter exceeds allowable value during ongoing observations, the BIM allows rearranging the structure or making suggestions concerning structure reinforcement on the fly. Thus, BIM became a crucial element of geospatial monitoring. Everyone gets benefits depending on their tasks. So, the main question is how to embed BIM into the standard process of geospatial monitoring and its workflow? The answer to this question aims at the given paper. The paper is divided into five sections. Section two provides an overview of the traditional approach to the geospatial monitoring concept. Section three deals with a modern idea of geospatial monitoring with BIM integration. Section four addresses the contemporary concept of geospatial monitoring for high-rise building monitoring, and section five outlines conclusions.

\section{GEOSPATIAL MONITORING: TRADITIONAL APPROACH}

The classical monitoring scheme is bound to the measured deformation characteristics, consequently dividing the observation methods depending on measured parameters. The measurement scheme is strongly dependent on the displacement type. Vertical displacements are being determined by spirit, trigonometric, or hydrostatic leveling. The horizontal displacements are being determined by linear or angular measurements and their combinations (triangulation, trilateration, traverses), GNSS. The deviation from vertical is monitored by the methods of directions, small angles, coordinates. The methods that allow determining the spatial displacements are close-range photogrammetry, GNSS, 
terrestrial laser scanning, space, ground-based interferometry, etc. The data volume is pretty extensive, and the complex analysis runs into some problems. The processing and simulation strategy is mainly limited to static models primarily based on polynomial regression. Despite various methods, the whole monitoring workflow can be boiled down to three main steps: design and first cycle measurements, outgoing measurements and displacements calculation, displacements analysis (Fig. 1).

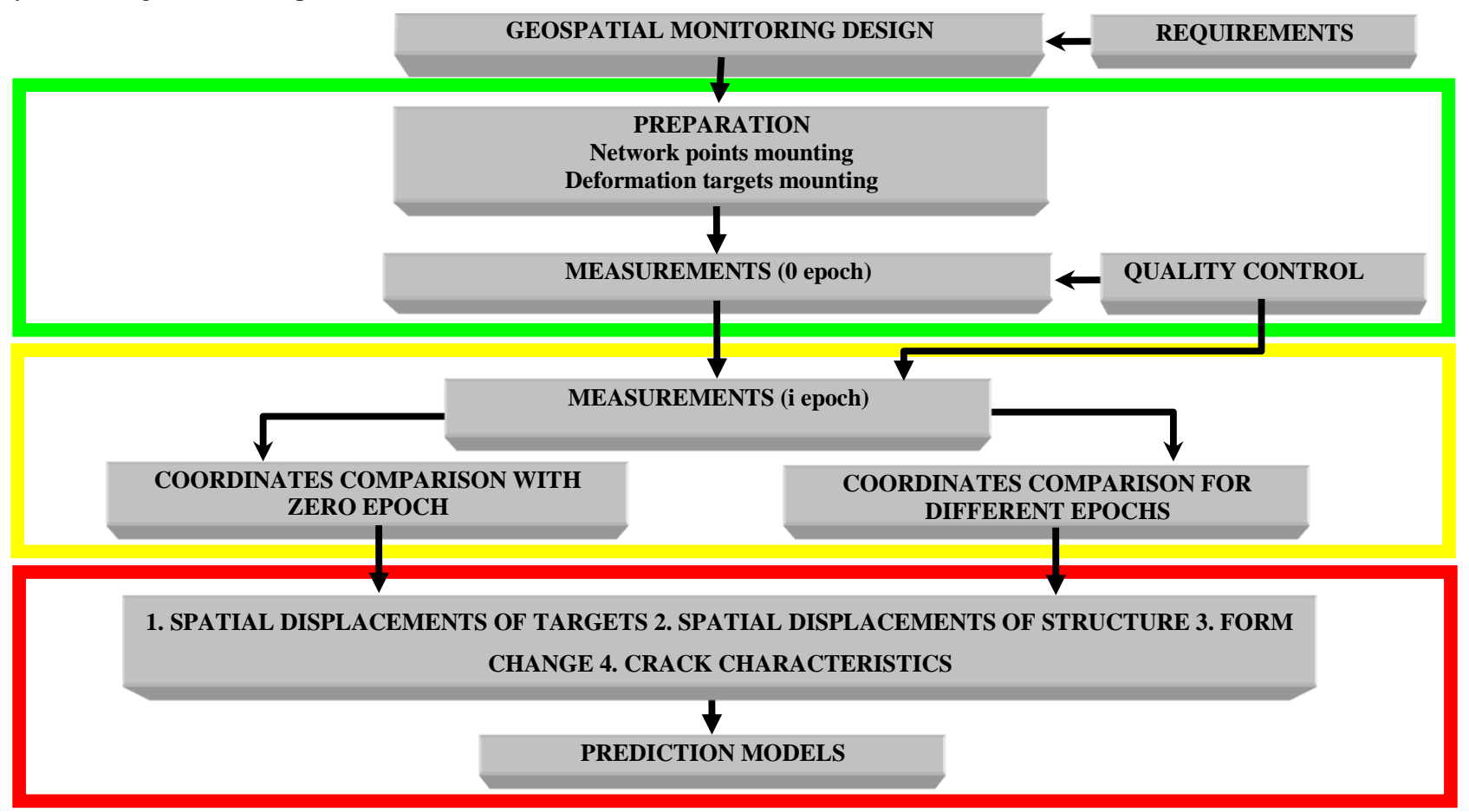

Figure 1. Workflow scheme of geospatial monitoring.

Geospatial monitoring design embraces network design, design of monitoring targets emplacement, preliminary accuracy calculation based on some external requirements or apriori knowledge, necessary equipment choice, and observation scheme design. The first observation, called zero epoch, is being carried out upon completing all these steps. The critical step is data quality check, namely their accuracy, reliability, and completeness. At the second stage, the measurements are accumulated, and after each epoch, the absolute coordinate differences according to zero epoch and relative coordinate differences regarding each other are calculated. Of course, a quality check is also applied during each observation epoch. The calculated differences are sent over to the third stage to calculate multiple characteristics of a structure deformation process (Fig. 1). Geospatial monitoring ends up with a prediction model generation. Depending on the task, various models can be constructed. One may find their description and pros and cons characteristics in geodetic literature. The described above workflow is separated from BIM. So, geospatial monitoring is being conducted in such a way that a structure digital model, a stage of a structure life cycle, and a structure purpose are not being taken into account. Such an approach may lead to disruption in results analysis and misleading comprehension of the structure deformation reasons. The wrong decision concerning the reparation or reinforcement work may be accepted in what follows. It is mandatory to include BIM in the geospatial monitoring workflow to overcome the problems and close the gaps between mentioned above issues.

\section{GEOSPATIAL MONITORING: MODERN CONCEPT}

Understanding the problems of traditional monitoring has led to the new modern concept of geospatial monitoring that is strongly related to BIM. Before considering the workflow of modern geospatial monitoring, it is worth comprehending the liaison between a building life cycle, BIM, and geospatial monitoring. A reasonable interpretation of this relationship gives a diagram in Fig. 2.

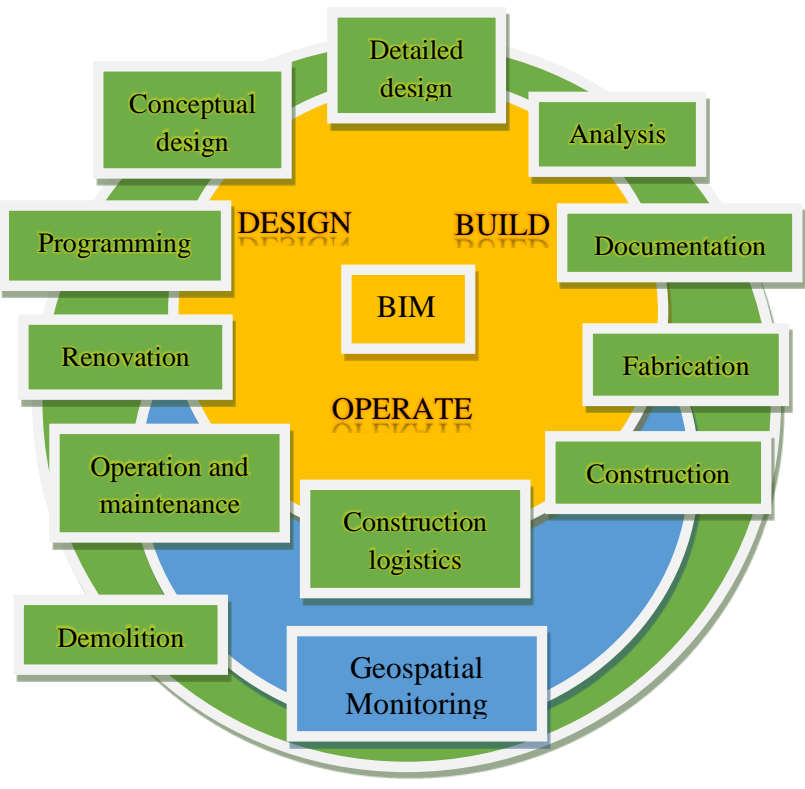

Figure 2. A liaison of Building Lifecycle, BIM, and Geospatial Monitoring.

As one may see, a building life cycle region includes BIM as a tool to support decisions during the building design, construction, and operation. At the same time, geospatial monitoring embraces the following stages of the building life cycle, namely, construction fabrication, structure assemblage, operation, 
renovation, and/or demolition. Evidently, geospatial monitoring is related to a building life cycle via BIM throughout these stages. Now, it is necessary to figure out the role of BIM at each stage of geospatial monitoring. First, let's present the suggested modern workflow and give the required description after that.

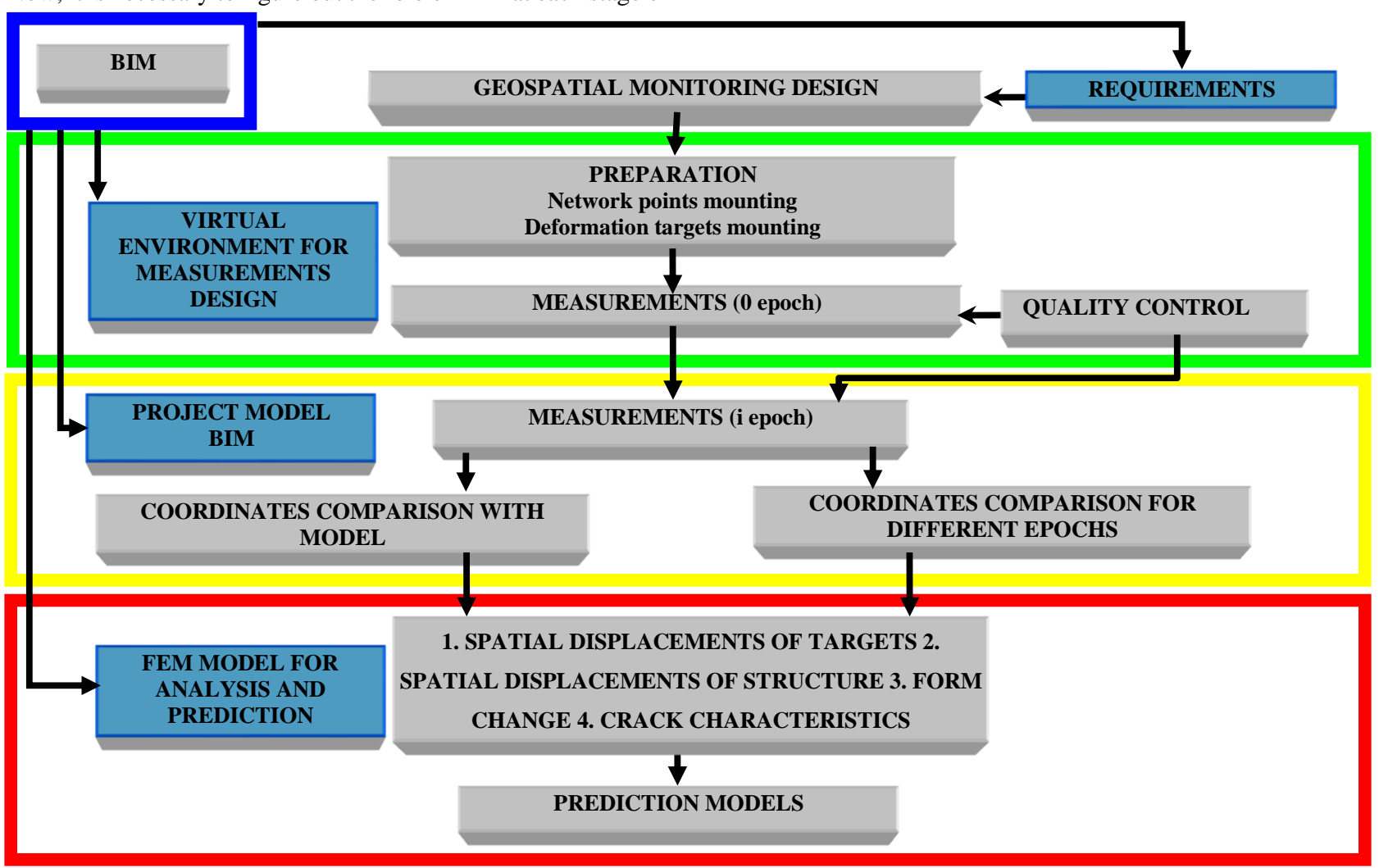

Figure 3. Workflow scheme of geospatial monitoring with BIM support.

BIM has appeared at the top of the workflow. It infers that BIM affects all stages of geospatial monitoring. The design stage needs BIM data to assign the observation accuracy correctly. The requirements must be drawn based on factual information about a structure without any premises or assumptions. That was the first drawback of traditional monitoring workflow. Thanks to $\mathrm{BIM}$, the requirements can be suggested by civil engineers or even derived by surveyors grounded on analytical and calculation models of the structure. Having the necessary accuracy, one may embark on the next step is designing the geodetic network and observation scheme. Here BIM creates a fascinating tool for design in the form of the virtual environment. It is well-known that field reconnaissance is the best solution for observation design. However, the virtual environment gives one the excellent opportunity to create a preliminary observation scheme that may be slightly refined during the field reconnaissance. On the other hand, virtual environmental makes possible the observation design with simultaneous network preanalysis. Therefore, a surveyor may create different variants, analyze the accuracy of those variants, and use the field check to validate the final observation scheme. As a result, the time for design and chance to make a wrong decision is cutting down significantly.

At the second step, observation collection, BIM suggests a new data source for monitoring. Except for comparing point coordinates for different epochs, the surveyor gets a chance to compare its results with an initial geometrical model. The task is simple if one has a set of targets with coordinates on the structure. But, if the mission is to compare, e.g., two separated in time point clouds generated by terrestrial laser scanning, the initial geometrical model can facilitate the analysis process. It becomes possible to compare the point cloud with the CAD model or solid model and reveal the features that can be hidden or distorted during the obvious cloud-to-cloud comparison. The same is valid for the models generated by close-range photogrammetry measurements.

Probably, the most valuable role BIM plays for the third stage where it deals with results analysis and prediction model creation. BIM gives a possibility to find a solution to the issue that one could not imagine twenty years ago. It is well-known that BIM comprises a bunch of models, e.g., architectural, analytical, calculation or numerical, and so on. Today, the calculation model for most tasks is presented as the FEM model. Such a model allows calculating all the essential parameters to wit moments, forces, shears, stresses, and so on under specific loads with any accuracy. Thanks to FEM, one may resolve an inverse issue. Let's suppose that we have determined displacements. For the FEM model, it is possible to upload the actual structure displacements and make an inverse simulation. FEM model will reflect the critical stresses in the structure elements and indicate the places that need the special attention of civil engineers. On the other hand, the FEM model allows predicting the structure behavior for the case of displacement growth. That predicting model will be the most precise because based on a physical model of the structure. So today, a surveyor uploads the displacements to BIM and instantaneously obtains the complex deformation picture. Now the surveyor may correct the observation scheme on-site, increase or decrease observation accuracy and add additional targets to the places with extreme stresses. Whereas a civil engineer may assess the structure state and determine whether the measured displacements lead to unallowable stresses in the structure elements or not. The development of prediction models for target displacements is still mandatory, but FEM makes this process a no-brainer and more effective.

Let's consider how the concept of modern geospatial monitoring works for hands-on case monitoring of high-rise buildings. 


\section{MODERN CONCEPT: A CASE STUDY OF HIGH- RISE BUILDING MONITORING}

The subject of the study is a typical twenty-three-story high-rise building. Commencing the design stage, the architectural model (Fig. 4) can be used for a general overview of the monitoring task.

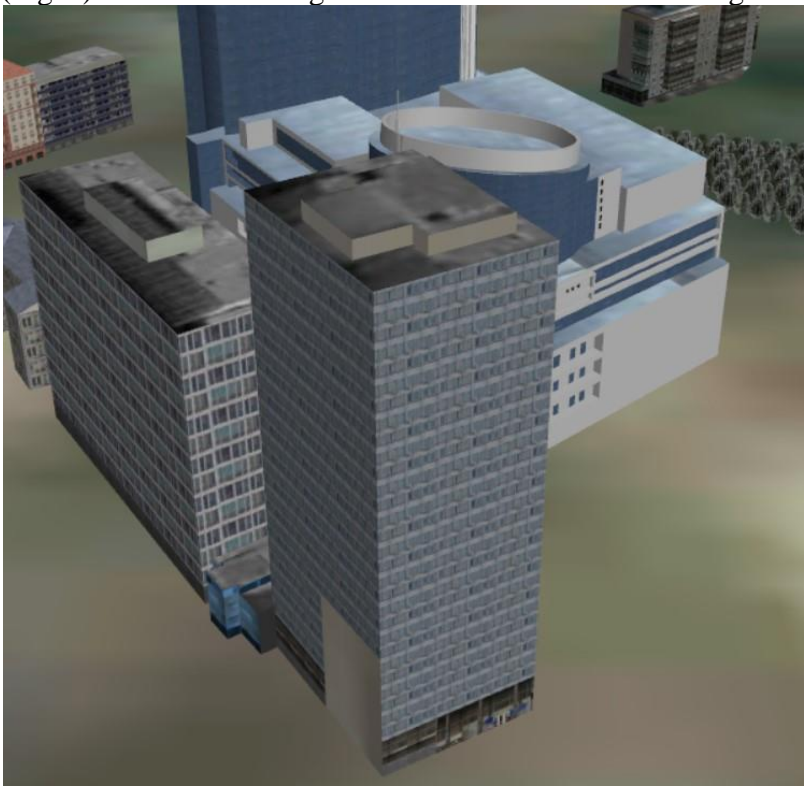

Figure 4. Architectural model.

The virtual model, generated from BIM, contains the structure itself and surrounding buildings with infrastructure (Fig. 5). A surveyor can conduct virtual reconnaissance to emplace the network points and observation targets.

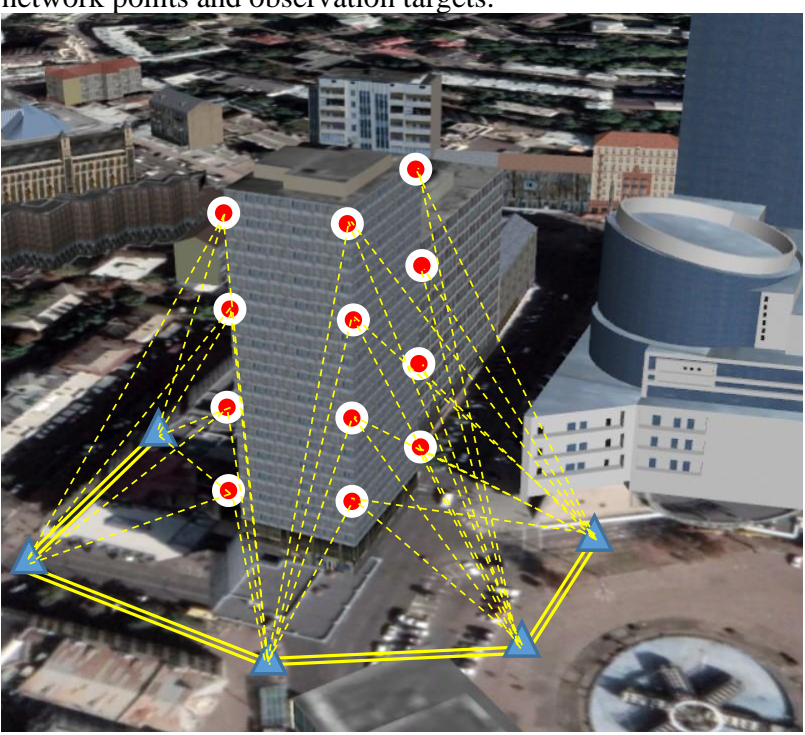

Figure 5. Virtual model for monitoring design and observation scheme.

Keeping in mind that this virtual model is already referenced to some external coordinate frame, one may use the software that instantaneously calculates the measured values (angles, distances, etc.) between network points and observation targets. Therefore, everything is ready for network and observation scheme preliminary analysis. That analysis typically employs a least squares adjustment procedure. A surveyor may create multiple schemes and analyze them, variating observation accuracy, types, and a number of observations. The software may resolve very complicated problems if the surrounding buildings and infrastructure are given as 3D models. One such issue is the intervisibility analysis using computational geometry methods. The software may calculate whether the optical ray from the observation station intersects some structures on its way or not. That problem sometimes cannot be resolved even upon field reconnaissance. After the final adoption of the observation scheme, everything is ready for zero observation epoch.

As it was mentioned before, BIM is a source of the various models (Fig. 6).
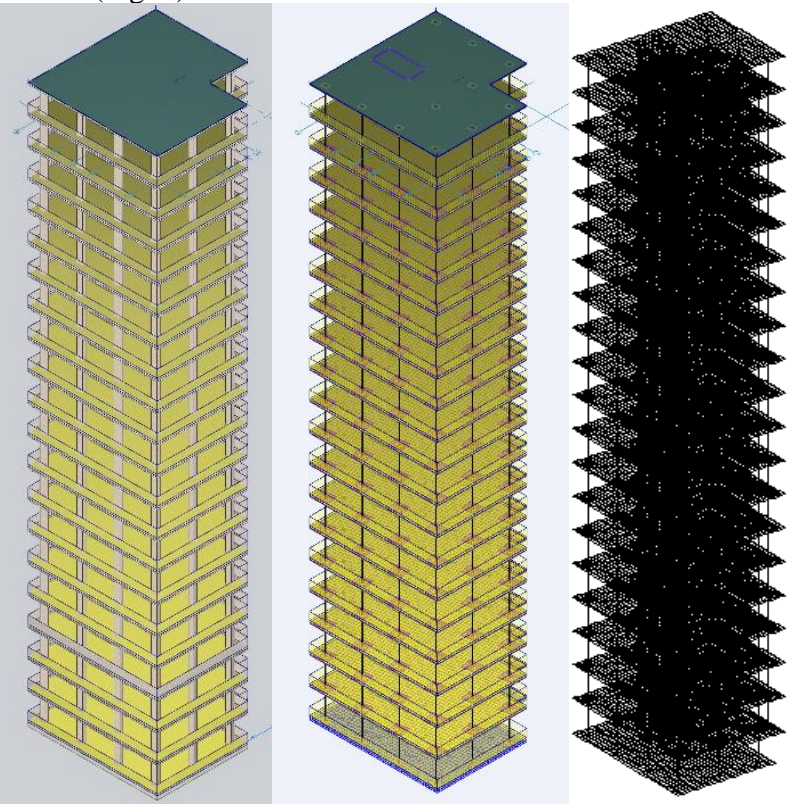

Figure 6. Various models are drawn from BIM: analytical, calculation, and meshed.

At the stage of data collection and coordinate differences calculation, the primary role plays the analytical model (Fig. 6). That model bears the main geometrical parameters that must be controlled during monitoring. It is worth emphasizing that the analytical model has to be generated by the results of as-built surveying. Thus, comparing the analytical model with measurement results, one obtains a crisp picture of the structure behavior between different epochs and from the moment the structure has been assembled.

The last but not least stage is displacements analysis and prediction.

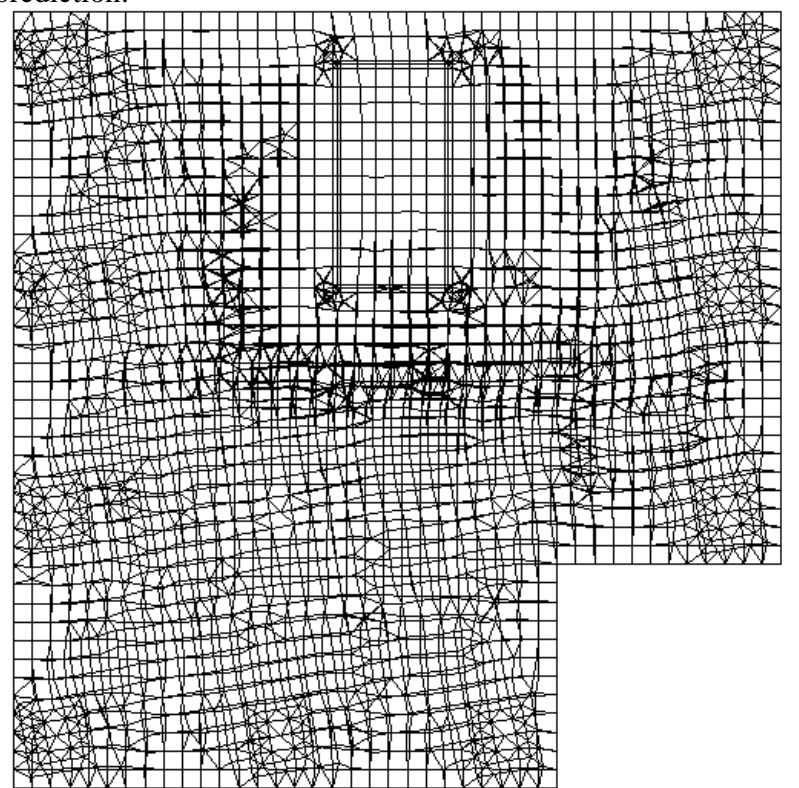

Figure 7. Zoomed meshed model for FEM analysis (top view). 
The crucial role at this stage acts the FEM model. This model is being generated from the calculation model using various meshing approaches (Fig. 6). Typically, the FEM model consists of linear elements for columns, surface (plane) triangular elements for non-massive slabs or shells, and volume elements for large slabs, e.g., basement slab.

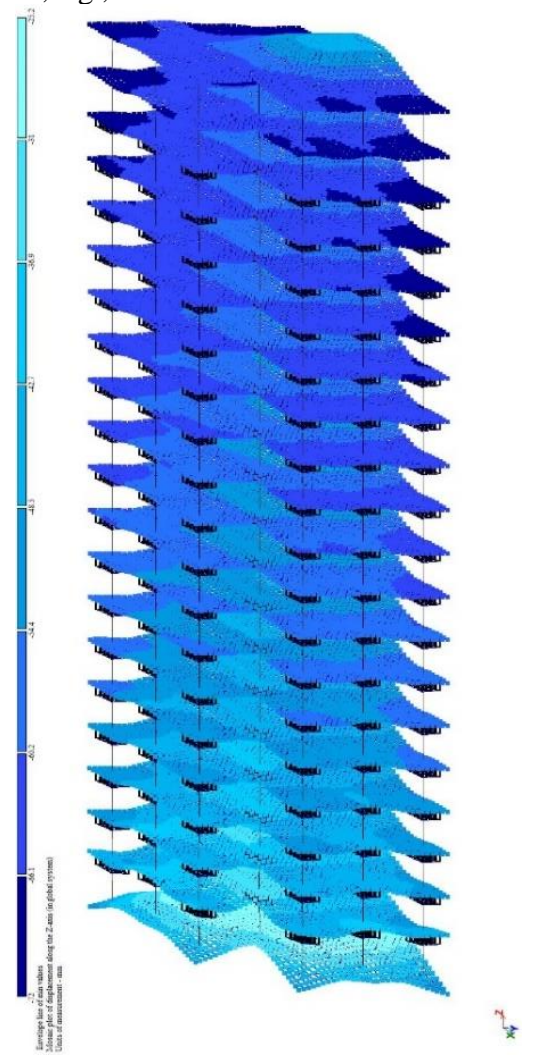

Figure 8. Displacement field along the $\mathrm{Z}$ axis in $\mathrm{mm}$.

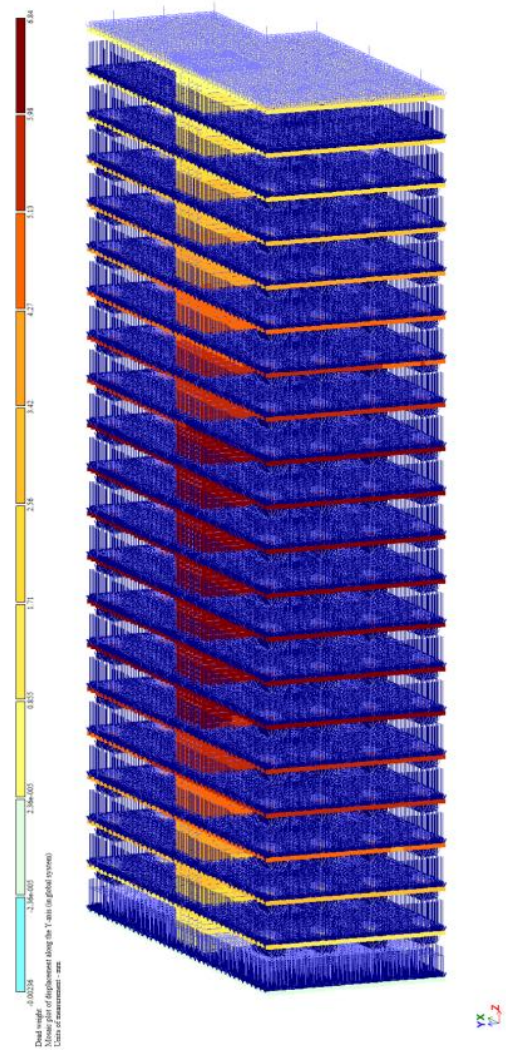

Figure 9. Displacement field along the $\mathrm{X}$ axis in $\mathrm{mm}$.

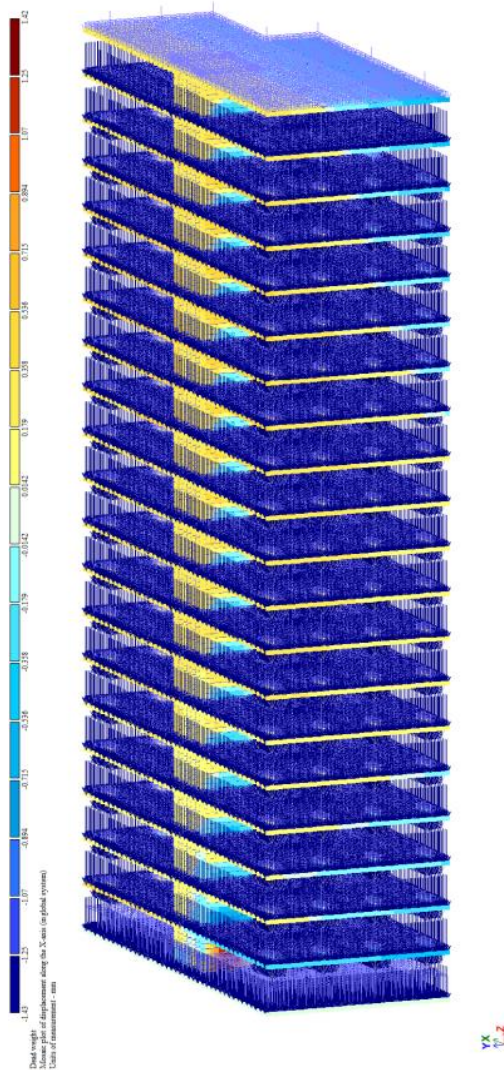

Figure 10. Displacement field along the $\mathrm{Y}$ axis in $\mathrm{mm}$.

The meshed model for the considered building is given in Fig. 6 . Fig. 7 portrays the top view of the meshed model where one may notice the triangular and quadrilateral shape of plane finite elements. BIM lets to carry out two types of displacements analysis. If we have actual loads and design loads, then the deformed models of the structure can be calculated. Then these models can be readily compared with each other and with the results of geospatial monitoring. If the structure operation conditions are not changed significantly during the operation stage, then the measured displacements should coincide with calculated displacements within some limits. These limits must be assigned during monitoring design. As a case study, the displacements along the coordinate axis are presented in Fig. 8. - Fig. 10. Also, the analysis can be performed for actual displacements. These displacements are being uploaded to the FEM model, and the loads are calculated. For that case, a surveyor and a civil engineer work together to find the places with extreme stresses and assign more frequent and precise monitoring for them. It is worth notifying that in both cases, the FEM model serves as a prediction model simultaneously. Of important to emphasize that the FEM model is already embedded into BIM, so a surveyor does not need any special skills to make the simulation. All that is necessary is to upload the displacements to BIM and get an output for further analysis.

\section{CONCLUSIONS}

The presented paper addressed the issue of the new concept development of geospatial monitoring of engineering structures. It was shown the relationship between a building life cycle, BIM, and geospatial monitoring. Based on this relationship, the obsolete geospatial monitoring workflow was analyzed, and the new one suggested, and BIM was determined as a cornerstone of the modern geospatial monitoring concept. The role of BIM for the design, data collection, and analysis were considered. Based 
on these results, the new concept of geospatial monitoring was applied for the monitoring of high-rise buildings. BIM creates a virtual environment for network and observation scheme preliminary analysis at the monitoring design stage. At the stage of data collection and coordinate differences calculation, the analytical model generated from BIM makes it possible to compare that model with measurement results and obtain a picture of the structure behavior between different epochs. Besides that, BIM lets to carry out two types of displacements analysis using FEM.

The paper has been presented a general view and approach to the claimed problem. Future studies will have to concentrate on indepth research on the relationship between BIM and geospatial monitoring for different building life cycle stages. Yet, BIM opportunities are not investigated in detail regarding the geospatial monitoring workflow and its features.

\section{REFERENCES}

Barazzetti, L., Banfi, F., Brumana, R., Gusmeroli, G., Oreni, D., Previtali, M., Roncoroni, F., Schiantarelli, G., 2015: BIM from laser clouds and finite element analysis: combining structural analysis and geometric complexity. Int. Arch. Photogramm. Remote Sens. Spatial Inf. Sci., XL-5/W4, 8103-8120. http://dx.doi.org/10.5194/isprsarchives-XL-5-W4-345-2015.

Crespi, P., Franchi, A., Ronca, P., Giordano, N., Scamardo, M., Gusmeroli, G., Schiantarelli, G., 2016: From BIM to FEM: the analysis of an historical masonry building. WIT Transactions on the Built $\quad$ Environment.
https://doi.org/10.2495/BIM150471.

Li, H.-N., Yi, T.-H., Yi, X.-D., Wang, G.-X., 2007: Measurement and analysis of windinduced response of tall building based on GPS technology. Advances in Structural Engineering 10(1): 8393.

Qin, G., Zhou, Y., Hu, K., Han, D., Ying, C., 2021: Automated Reconstruction of Parametric BIM for Bridge Based on Terrestrial Laser Scanning Data. Advances in Civil Engineering 2021. https://doi.org/10.1155/2021/8899323

Shults, R., 2020: The Models of Structural Mechanics for Geodetic Accuracy Assignment: A Case Study of the Finite Element Method. In Contributions to International Conferences on Engineering Surveying, 187-197. https://doi.org/10.1007/9783-030-51953-7_16

Tang, P., Huber, D., Akinci, B., Lipman, R., Lytle, A., 2010: Automatic reconstruction of as-built building information models from laser-scanned point clouds: A review of related techniques. Automation in Construction 19: 829-843. https://doi.org/10.1016/j.autcon.2010.06.007

Xu, W., Xu, X., Yang, H., Neumann, I., 2019: Optimized finite element analysis model based on terrestrial laser scanning data. $\begin{array}{llll}\text { Composite } & \text { Structures } & \text { 207: } & \text { 62-71. }\end{array}$ https://doi.org/10.1016/j.compstruct.2018.09.006 